Astrophysics and Space Science manuscript No.

(will be inserted by the editor)

E. V. Gotthelf · J. P. Halpern

\title{
The Anatomy of a Magnetar: XMM Monitoring of the Transient Anomalous X-ray Pulsar XTE J1810-197
}

Received: date / Accepted: date

\begin{abstract}
We present the latest results from a multiepoch timing and spectral study of the Transient Anomalous X-ray Pulsar XTE J1810-197. We have acquired seven observations of this pulsar with the Newton X-ray Multi-mirror Mission (XMM-Newton) over the course of two and a half years, to follow the spectral evolution as the source fades from outburst. The spectrum is arguably best characterized by a two-temperature black' body whose luminosities are decreasing exponentially with $\tau_{1}=870 \mathrm{~d}$ and $\tau_{2}=280 \mathrm{~d}$, respectively. The temperatures of these components are currently cooling at a rate of $22 \%$ per year from a nearly constant value recorded at earlier epochs of $k T_{1}=0.25 \mathrm{keV}$ and $k T_{2}=0.67 \mathrm{keV}$, respectively. The new data show that the temperature $T_{1}$ and luminosity of that component have nearly returned to their historic quiescent levels and that its pulsed fraction, which has steadily decreased with time, is now consistent with the previous lack of detected pulsations in quiescence. We also summarize the detections of radio emission from XTE J1810-197, the first confirmed for any AXP. We consider possible models for the emission geometry and mechanisms of XTE J1810-197.
\end{abstract}

Keywords pulsars: general — stars: individual (XTE J1810-197) — stars: neutron — X-rays: stars

PACS 95.85.-e · 95.85.Nv · 97.60.Jd · 97.60.Gb . 95.75

XMM-Newton is an ESA science mission with instruments and contributions directly funded by ESA Member States and NASA. This research is supported by XMM-Newton grant NNG05GJ61G and NASA ADP grant ADP04-0059-0024.

E. V. Gotthelf

Columbia Astrophysics Laboratory, Columbia University, 550

West $120^{t h}$ Street, New York, NY 10027-6601

E-mail: eric@astro.columbia.edu

\section{Introduction}

Neutron star (NS) astronomy has been recently invigorated by the identification of a new class of magnetically dominated emitters. Known as anomalous X-ray pulsars (AXPs) and soft gamma-ray repeaters (SGRs), these objects are apparently young, isolated neutron stars (NSs), whose properties differ markedly from those of the Crab pulsar, previously considered prototypical of the young NSs (for a review see Mereghetti et al. 2002). All AXPs and at least 3 of the 4 SGRs are identified as relatively slow $(5-12 \mathrm{~s})$ pulsars. Many are located at the centers of recognized, young supernova remnants (SNRs), directly associating them with their supernova explosions. These objects emit predominantly at X-ray energies and are distinguished by their characteristic spectral signature. This radiation cannot be accounted for by rotational energy losses alone, as for the radio pulsars, but is most likely powered by the decay of an enormous magnetic field characterized by a dipole with $B_{p} \gtrsim 4.4 \times$ $10^{13} \mathrm{G}$, at the pole. Collectively, these isolated NSs are understood within the context of the magnetar theory (Duncan \& Thompson 1992; Thompson \& Duncan 1996).

A unique pulsar has been discovered whose remarkable properties offer great promise for deciphering the emission mechanism(s) of magnetars. XTE J1810-197 is a $5.54 \mathrm{~s}$ X-ray pulsar whose measured and derived physical parameters are fully characteristic of an AXP, but expresses behavior not previously associated with any such object (Gotthelf et al. 2004, hereafter Paper I). XTE J1810-197 is a transient AXP (TAXP) - it was discovered during a bright impulsive outburst (Ibrahim et al. 2004) that is still fading steadily. Even more surprising is the discovery of highly variable radio emission, providing the first confirmed example of radio flux from an AXP (Halpern et al. 2005b), and the subsequent detection of radio pulsations at the X-ray period (Camilo et al. 2006).

The outburst that resulted in the detection of the transient AXP occurred sometime between 2002 November and 2003 January (Ibrahim et al. 2004). Since then, 
E. V. Gotthelf, J. P. Halpern

over the course of a year, regular scans of the region with RXTE recorded an exponential flux decay with a time-constant of $269 \pm 25$ days from a maximum of $F(2-10 \mathrm{keV}) \approx 8 \times 10^{-11} \mathrm{erg} \mathrm{cm}^{-2} \mathrm{~s}^{-1}$. In comparison, the previous average quiescent flux, with its softer spectrum, gives $F(0.5-10 \mathrm{keV}) \approx 5.5 \times 10^{-13} \mathrm{erg} \mathrm{cm}^{-2} \mathrm{~s}^{-1}$ (Paper I). This contrast is unprecedented for an AXP. Further RXTE observations yielded SGR-like episodes of bursts (Woods et al. 2005), similar to those seen from 1E 2259+586 (Kaspi et al. 2003). A search for an optical/IR counterpart detected a fading IR source within the Chandra error circle, similar to ones associated with other AXPs, confirming its identification with XTE J1810-197 (Rea et al. 2004a b).

In this paper we present the latest results from TAXP XTE J1810-197 including new XMM-Newton observations extending to 2006 March. These observations allow us to characterize the spectral evolution of a TAXP in outburst as it returns to quiescence. We show that the spectrum has now begun a marked transition back to the nominal quiescent state, with a distinct temperature and flux evolution. This long-term evolution provides the unique possibility of decomposing its fading spectral components in a manner unavailable for other magnetars (Halpern \& Gotthelf 2005, hereafter Paper II). In the following, we used a revised distant to the NS, $d_{3.3}$, quoted in units of $3.3 \mathrm{kpc}$ and based on the radio pulsar DM measurement of Camilo et al. (2006).

\section{Long-term spectral and temporal evolution}

Herein we present a total of seven XMM-Newton observations of XTE J1810-197 of which four have been previously described in Papers I, II, and Gotthelf \& Halpern (2005, hereafter Paper III). The three new data sets were obtained using similar observing modes as for previous observations and reduced and analyzed in an identical manner. A log of these observations is recorded in Table 1. A full report on the reduction and analysis of these data sets will be presented in Gotthelf et al. (in prep.).

Figure 1 presents an up-to-date light curve of TAXP XTE J1810-197 derived by adding the XMM-Newton flux measurements to the RXTE results of Ibrahim et al. (2004). For comparison, the XMM-Newton fluxes were extracted assuming a simple power-law spectral model fitted in the $2-10 \mathrm{keV}$ energy band. The combined data points are well fitted by an exponential decay model with overall time-constant of $233.5 \mathrm{~d}$, somewhat shorter but consistent with the initial RXTE trend, given the extended monitoring interval. To allow for a systematic offset in the RXTE flux measurements ${ }^{1}$, these data were rescaled by a factor of 1.42 to match the XMM-Newton results. The implied flux range for the initial outburst is

\footnotetext{
1 Ibrahim et al. (2004) assumed a nominal $2-10 \mathrm{keV}$

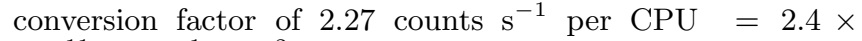
$10^{-11} \mathrm{erg} \mathrm{s}^{-1} \mathrm{~cm}^{-2}$.
}

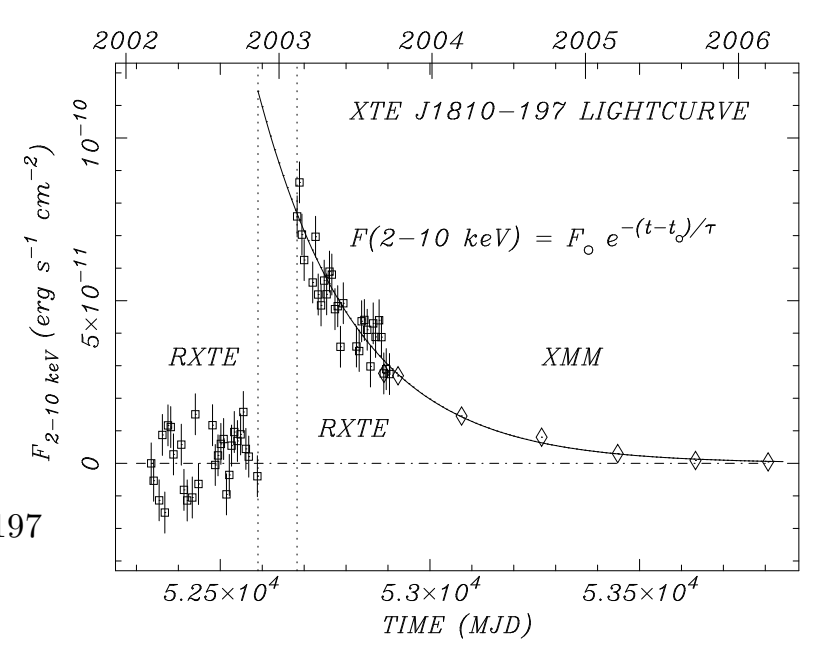

Fig. 1 Long-term light-curve of XTE J1810-197 using measurements obtained with RXTE (squares) and XMM-Newton (diamond). The RXTE data are from Ibrahim et al. (2004), renormalized to match the XMM-Newton results. The XMMNewton data points are for the observations of Table 1, but fitted with a power-law model in the $2-10 \mathrm{keV}$ energy band, for direct comparison with the RXTE results. The solid line is a best combined fit to an exponential decay model (see text for parameters).

$F(2-10 \mathrm{keV})=(11-8) \times 10^{-11} \mathrm{erg} \mathrm{s}^{-1} \mathrm{~cm}^{-2}$, over the interval during which the target was inaccessible to RXTE. Clearly the flux in this band has all but returned to its pre-outburst value.

Spectra of AXPs are nominally modeled assuming a two-component power-law plus blackbody model. The spectral and temporal trends for XTE J1810-197 allow us to strongly reject this model (see Paper II for a comprehensive discussion). Instead, we find that a twotemperature blackbody model gives equally acceptable spectral fits but is better motivated physically (see $§ 5$ ). A summary of spectral results is presented in Table 1 and the spectra, fitted with the double blackbody model, are displayed in Figure 2] (the 2003 Oct observation is excluded here for clarity). Although the hot blackbody component (BB2; Fig. 3) initially dominated the emission at low energies, it fades more rapidly than the cooler "warm" emission component (BB1; Fig. 3). Evidently the spectral fits after 2004 March deviate significantly at the lower end of the XMM-Newton energy band, below $0.7 \mathrm{keV}$. It is not yet clear if this is an instrumental artifact, however, taken at face value there seems to be an absorption feature at $\approx 350 \mathrm{eV}$ with a Gaussian width of $\sigma \approx 160 \mathrm{eV}$. The mean equivalent width of this feature is $600 \mathrm{eV}$, but its strength is somewhat time dependent. Perhaps as the hotter component contributes less, this feature becomes more evident, suggesting an association exclusively with the cooler component. Further research is needed to quantify this feature and consider its reality, or physical implications.

The spectrum of XTE J1810-197 can be thought of as the combined flux from two concentric emitters, "hot 
Table 1 XMM-Newton Spectral Results for XTE J1810-197

\begin{tabular}{lccccccc}
\hline Parameter & 2003 Sep 8 & 2003 Oct 12 & 2004 Mar 11 & 2004 Sep 18 & 2005 Mar 18 & 2005 Sep 20 2006 Mar 18 \\
\hline Expo (ks) & $11.5 / 8.1$ & $6.9 / 6.2$ & $17.0 / 15.8$ & $26.5 / 24.4$ & $39.8 / 37.2$ & $39.5 / 37.8$ & $41.7 / 38.8$ \\
$N_{\mathrm{H}}\left(\mathrm{cm}^{-2}\right)^{b}$ & $0.65 \pm 0.04$ & $0.65 \pm 0.04$ & $0.65 \pm 0.04$ & 0.65 (fixed) & 0.65 (fixed) & $0.65($ fixed) & $0.65($ fixed) \\
$k T_{1}(\mathrm{keV})$ & $0.25 \pm 0.02$ & $0.29 \pm 0.04$ & $0.27 \pm 0.02$ & $0.25 \pm 0.01$ & $0.22 \pm 0.01$ & $0.20 \pm 0.01$ & $0.19 \pm 0.01$ \\
$k T_{2}(\mathrm{keV})^{2}$ & $0.68 \pm 0.02$ & $0.71 \pm 0.03$ & $0.70 \pm 0.01$ & $0.67 \pm 0.01$ & $0.60 \pm 0.01$ & $0.52 \pm 0.01$ & $0.46 \pm 0.02$ \\
$A_{1}\left(\mathrm{~cm}^{2}\right)$ & $5.6 \times 10^{12}$ & $2.9 \times 10^{12}$ & $3.3 \times 10^{12}$ & $4.0 \times 10^{12}$ & $4.9 \times 10^{12}$ & $6.6 \times 10^{12}$ & $7.2 \times 10^{12}$ \\
$A_{2}\left(\mathrm{~cm}^{2}\right)$ & $2.8 \times 10^{11}$ & $2.2 \times 10^{11}$ & $1.3 \times 10^{11}$ & $8.7 \times 10^{10}$ & $6.0 \times 10^{10}$ & $3.7 \times 10^{10}$ & $3.6 \times 10^{10}$ \\
$\mathrm{BB} 1 \mathrm{Flux}^{c}$ & $4.2 \times 10^{-12}$ & $5.4 \times 10^{-12}$ & $3.5 \times 10^{-12}$ & $2.6 \times 10^{-12}$ & $1.6 \times 10^{-12}$ & $1.0 \times 10^{-12}$ & $7.5 \times 10^{-13}$ \\
$\mathrm{BB} 2 \mathrm{Flux}^{c}$ & $3.5 \times 10^{-11}$ & $3.0 \times 10^{-11}$ & $1.8 \times 10^{-11}$ & $1.0 \times 10^{-11}$ & $4.0 \times 10^{-12}$ & $1.3 \times 10^{-12}$ & $6.8 \times 10^{-13}$ \\
Total Flux $^{c}$ & $3.93 \times 10^{-11}$ & $3.84 \times 10^{-11}$ & $2.13 \times 10^{-11}$ & $1.29 \times 10^{-11}$ & $5.67 \times 10^{-12}$ & $2.35 \times 10^{-12}$ & $1.44 \times 10^{-12}$ \\
$L_{\mathrm{BB} 1(\mathrm{bol})^{d}}$ & $2.4 \times 10^{34}$ & $2.3 \times 10^{34}$ & $1.7 \times 10^{34}$ & $1.6 \times 10^{34}$ & $1.2 \times 10^{34}$ & $1.0 \times 10^{34}$ & $8.6 \times 10^{33}$ \\
$L_{\mathrm{BB} 2(\mathrm{bol})^{d}}$ & $6.3 \times 10^{34}$ & $5.7 \times 10^{34}$ & $3.1 \times 10^{34}$ & $1.8 \times 10^{34}$ & $7.9 \times 10^{33}$ & $2.8 \times 10^{33}$ & $1.7 \times 10^{33}$ \\
$\chi_{\nu}^{2}($ dof $)$ & $1.1(187)$ & $1.1(84)$ & $1.1(194)$ & $1.2(188)$ & $1.6(152)$ & $1.6(80)$ & $1.6(117)$ \\
\end{tabular}

Note - Uncertainties in spectral parameters are 90\% confidence for two interesting parameters.

${ }^{a}$ EPIC-pn exposure/livetime in units of ks.

${ }^{b}$ Interstellar hydrogen absorbing column density in units of $\mathrm{cm}^{-2}$.

${ }^{c}$ Absorbed 0.5-10 keV flux in units of erg $\mathrm{cm}^{-2} \mathrm{~s}^{-1}$.

${ }^{d}$ Unabsorbed bolometric luminosity in units of erg $\mathrm{s}^{-1}$ assuming a distance of $d=3.3 \mathrm{kpc}$.

spots", whose temperature and size are evolving at different rates, effectively changing the overall shape of the spectrum with time (Paper II). With a set of spectral measurements spanning two and a half years, a clear trend has emerged. The bolometric luminosities of the two components are shown in Figure 3 This reveals a cooler component decreasing exponentially in time with $\tau_{1}=870 \mathrm{~d}$, while the hotter temperature flux declines with $\tau_{2}=280 \mathrm{~d}$. As might be expected, the shorter time constant is very close to the one described by the long-term flux above $2 \mathrm{keV}$ (Figure 1), where the hotter blackbody component dominates. However, the latest two data points show that this component has now fallen faster than the original exponential. Although the bolometric luminosities of the two components are quite different at the latest epoch, their measured fluxes are now nearly equal in the $0.5-8 \mathrm{keV}$ range. We also note that, while it is possible to fit an alternative power-law decay model to the hot blackbody flux, such a model would require a decay index that steepens with time.

Based on the decay rates and approximate outburst time we estimate an initial bolometric luminosity for the two spectral components of $L_{1} \approx 3 \times 10^{34} d_{3.3}^{2} \mathrm{erg} \mathrm{s}^{-1}$ and $L_{2} \approx 2 \times 10^{35} d_{3.3}^{2} \mathrm{erg} \mathrm{s}^{-1}$, respectively. The total luminosity at the peak of the outburst is comparable to that of a persistent AXP, suggesting that this TAXP is anomalously faint. We can now compute the implied fluences of $f_{1} \approx 2 \times 10^{42} d_{3.3}^{2}$ erg and $f_{2} \approx 4 \times 10^{42} d_{3.3}^{2} \mathrm{erg}$, for the two components, respectively. The energy of the outburst event is many orders-of-magnitude lower than that for a typical SGR flare.

The temperatures and inferred blackbody emitting areas have also evolved measurably with time. Figure 4 displays the time histories of these parameters for each spectral component, obtained from the model fits. Based

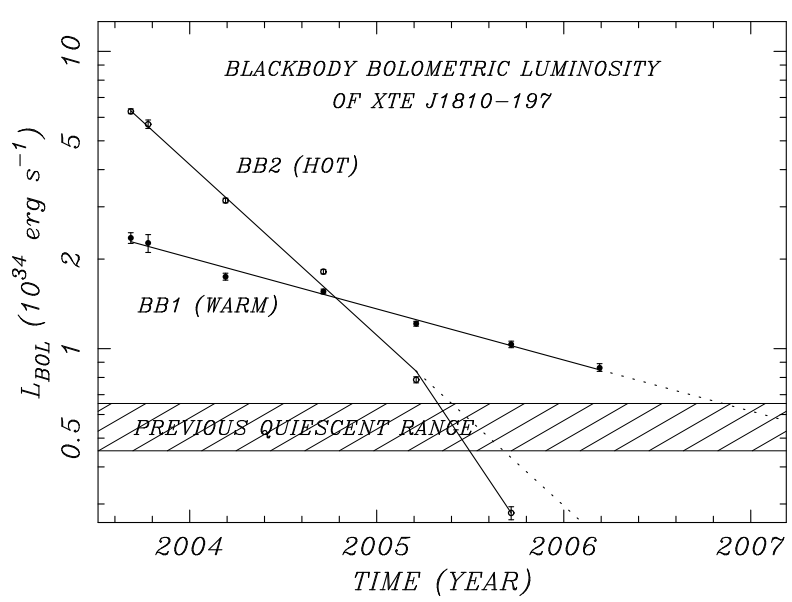

Fig. 3 A semi-log plot of the bolometric luminosity of XTE J1810-197 as a function of time (crosses) for each temperature component of the double blackbody model given in Table 1. The light-curves are fit assuming an exponential decays model (solid lines); the corresponding e-folding times is $\tau_{1}=280 \mathrm{~d}$ and $\tau_{2}=870 \mathrm{~d}$ for the warm and hot temperature components, respectively. The recent data for the hot component now deviates from this model. The fitted quantities have been extrapolated to the $(1 \sigma)$ quiescent range measured in Paper I (cross-hatched area).

on the three latest data points, the temperatures now show a definite cooling trend for each spectral component, as suggested by the broken line in Figure 4 Prior to mid 2004, the temperatures likely remained nearly constant, after which they both fell at a rate of $\approx 22 \%$ per year $\left(\Delta k T_{1}=-0.051 \mathrm{keV} \mathrm{yr}^{-1} ; \Delta k T_{2}=-0.15 \mathrm{keV} \mathrm{yr}^{-1}\right)$. The size of the effective hot spots (derived from the blackbody emission areas) also followed distinct evolutions. The hot component has been shrinking exponentially since the initial XMM-Newton observation, while 

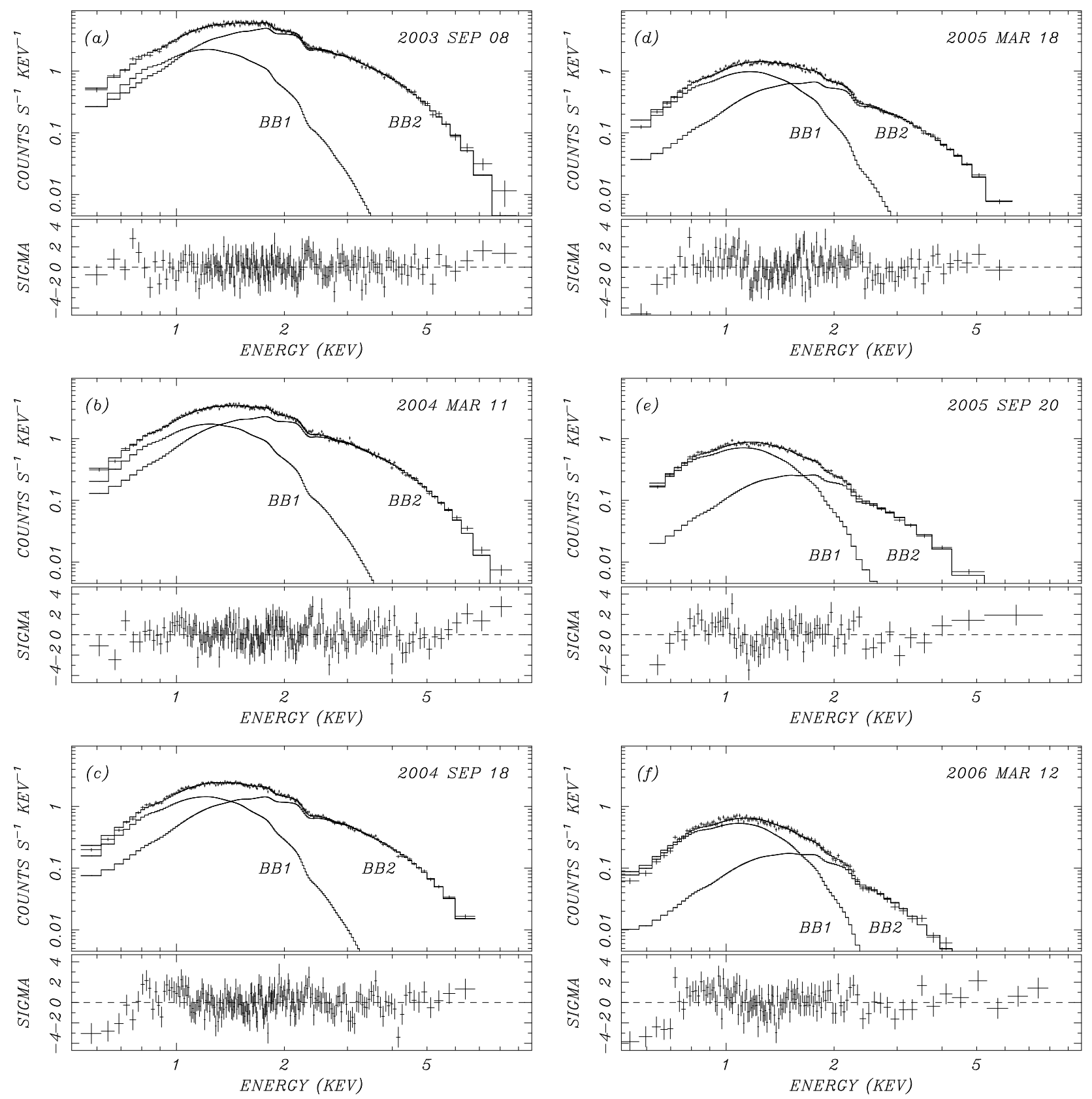

Fig. 2 XMM-Newton EPIC pn spectra of XTE J1810-197 from the earliest to the latest epochs, in six months intervals (the 2003 Oct spectrum is excluded, for clarity). These spectra are shown with the best-fit two-temperature blackbody model specified in Table 1. Although the temperatures of the blackbody components have not changed greatly between epochs, the flux of the hot component (BB2) has decayed rapidly relative to that of the cooler one (BB1). Also shown are the residuals from the best-fit models. The nature of the deviations to the model below $0.7 \mathrm{keV}$ has yet to be determined (see text).

the warm component has steadily increased in size. A notable exception is the initial data point which deviates from the trend for both the areas and temperatures. This may be associated with a glitch or rotation instability, as suggested by the pulse timing results around this epoch $(\S 3)$.

The area of the warm component $\left(A_{1}\right.$; Table 1$)$ may be reaching a maximum, corresponding to the whole NS surface. This would then provide a lower-limit on the
NS radius of $\gtrsim 7.5 d_{3.3} \mathrm{~km}$, ignoring relativistic effects (redshift and light bending). In contrast, the area of the hotter component continues to shrink and its contribution to the flux is severely diminished.

As shown in Figure 3, the X-ray luminosity of TAXP XTE J1810-197 has nearly returned to its historic quiescent level. This is also true of the temperature and inferred blackbody area. The pre-burst ROSAT spectrum of 1992 March 7 is reasonably well fitted with 

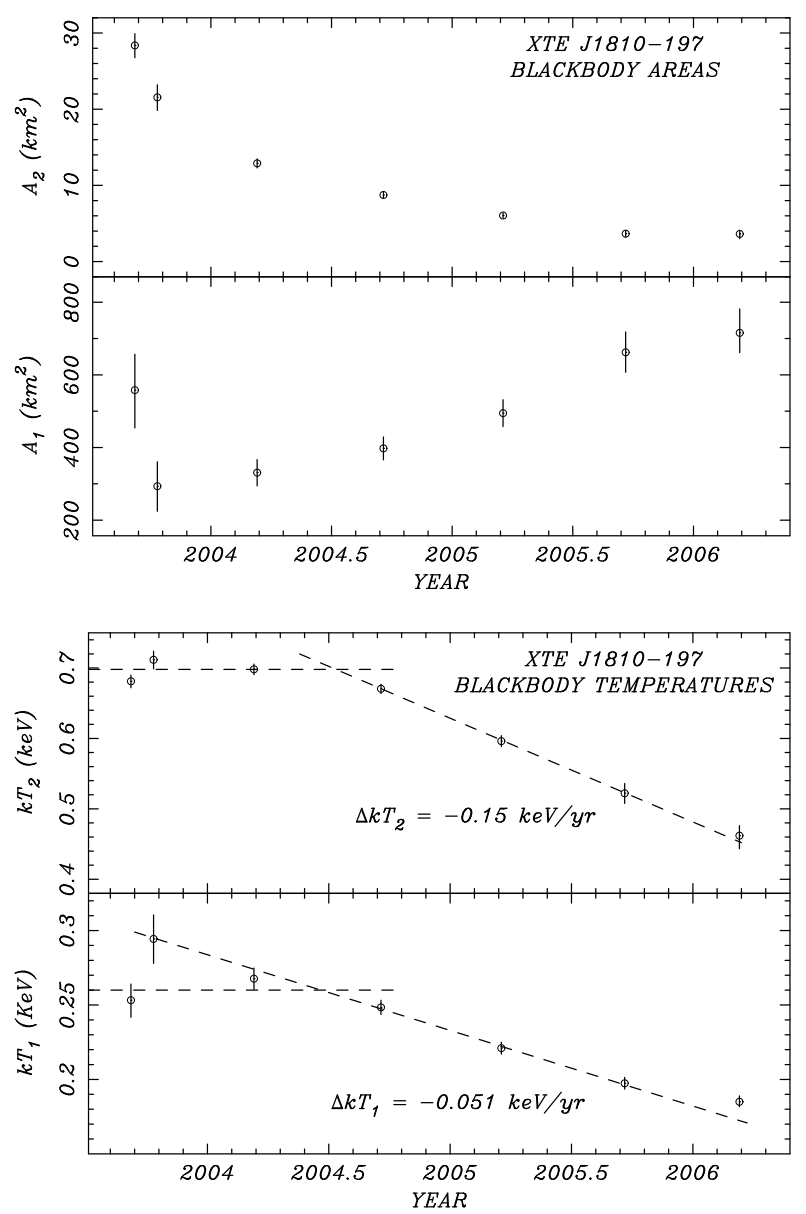

Fig. 4 The time history of the best-fit XMM-Newton component temperatures and surface emitting areas for XTE J1810-197 based on the double blackbody spectral model (Table 1). The evolving areas (Top) indicates that the warm component is expanding to cover the NS surface, while the hot component is shrinking rapidly. The corresponding temperatures (Bottom) are declining at a steady rate of $22 \%$ per year since mid 2004. Prior to that time the temperature of the hotter component was essentially constant. This trend for the warm component is somewhat ambiguous.

a single blackbody of $k T=0.18 \pm 0.02 \mathrm{keV}$ covering $5.2 \times 10^{12} d_{3.3}^{2} \mathrm{~cm}^{2}$ and $L_{B B}(\mathrm{bol})=5.6 \times 10^{33} d_{3.3}^{2} \mathrm{erg} \mathrm{s}^{-1}$ (Paper I). The latest XMM-Newton spectrum that overlaps the ROSAT band is again dominated by the cooler blackbody component. Furthermore, the current pulsed fraction is consistent with the quiescent upper-limits. Thus the double blackbody model is sufficient to describe the observed properties, and may not require a third "quiescent" component to explain the pre-outburst observations. XMM-Newton data from the next observing window (2006 September) is likely to be consistent with the ROSAT result in the soft energy band, effectively defining a return to the quiescence state.

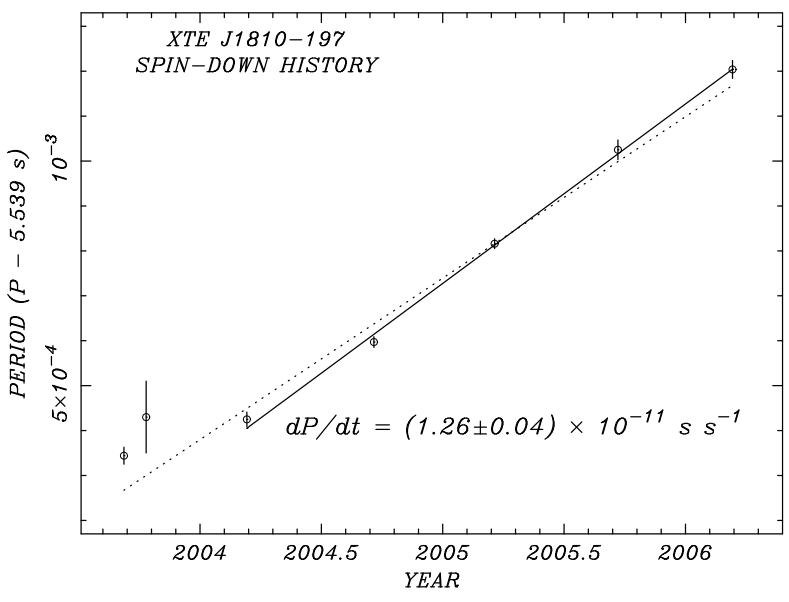

Fig. 5 The spin-down history of XTE J1810-197 pulse period as measured using the XMM-Newton data of Table 1. The initially erratic spin-down appears to be settling down the last 5 data points are well-modeled by a linear spin-down model (solid line); in contrast, a poor fit is obtained using all points (dotted line).

\section{Spin-down Evolution of XTE J1810-197}

The barycentered pulse period of XTE J1810-197 measured at each XMM-Newton observing epoch is shown in Figure [5] These data points were derived following the method outlined in Paper I. Prior to epoch 2004, the spin-down rate derived from the RXTE and initial XMM-Newton measurements were highly erratic, ranging from $\dot{P}=(0.8-2.2) \times 10^{-11} \mathrm{~s} \mathrm{~s}^{-1}$ Ibrahim et al. 2004; Paper III). This temporal behavior is likely associated with the outburst event. The latest period measurements suggest that the spin-down rate has settled down somewhat. A linear model fit to the last 5 period measurements yields a period derivative of $\dot{P}=$ $1.26 \pm 0.04 \times 10^{-11} \mathrm{~s} \mathrm{~s}^{-1}$ with a $\chi_{\nu}^{2}=1.2$ for $3 \mathrm{DoF}$, corresponding to a null hypothesis probability of 0.27 . Compared to the radio $\dot{P}=1.016 \pm 0.001 \times 10^{-11} \mathrm{~s} \mathrm{~s}^{-1}$ of 17 March - 7 May (Camilo et al. 2006), there is evidently still timing noise in the pulsar's spin-down. The revised X-ray rate implies a nominal characteristic age $\tau_{\mathrm{c}}=7.0 \mathrm{kyr}$, surface magnetic field $B_{\mathrm{s}}=2.7 \times 10^{14} \mathrm{G}$, and spin-down power $\dot{E}=2.9 \times 10^{33} \mathrm{erg} \mathrm{s}^{-1}$, values typical for a magnetar.

The energy-dependent modulation of the pulsar has evolved noticeably over time with the declining flux. Figure 6 displays the pulse profiles at each XMM-Newton epoch, folded at the best determined periods. The data has been broken up into six energy bands, with the corresponding background in each band subtracted. The pulsed fractions, defined as the pulsed emission divided by the total flux, are indicated on the corner of each panel of the figure. These profiles are aligned so that phase zero is the same at each epoch, for ease of comparison. In any case, within an observation, the phase zero does not change with energy. This suggests a geo- 
metric interpretation for the modulation, such as from localized emission on the rotating NS star surface. The pulsed fraction has generally decreased with time, most notably at X-ray energies below $E<2 \mathrm{keV}$; at higher energies the trend is less clear due to the large uncertainties derived for those pulsed fractions. Furthermore, at all epochs, the pulsed fraction clearly increases with energy, an equally interesting result discussed below.

The evolving spectral shape and pulse profiles of TAXP XTE J1810-197 provide an important and unique (so far) diagnostic of the emission geometry, and ultimately, the emission mechanism(s) of NSs. Because the spectrum is well modeled by two blackbody components, and the phase alignment of the pulse profiles are energy independent, it is most natural to consider emission from two concentric regions on the NS surface. The hot component is associated with a smaller hot-spot, while the blackbody model predicts a larger annulus for the warm component. The complete collection of observed pulsed fractions is consistent with this model. The smaller, hotter spot always dominates the spectrum above $2 \mathrm{keV}$ and offers a natural explanation for the higher modulation at these energies. However, at lower energies, this contribution gradually fades relative to cooler emission (which is less modulated), contributing less and less to the pulsed emission over time.

We note in passing that a fitted power-law spectral component would have to dominate the soft $(<2 \mathrm{keV}) \mathrm{X}$ rays at all epochs, while varying in its contribution to the hard $(>2 \mathrm{keV}) \mathrm{X}$-rays. Such a power law would drive an evolution of the pulse shapes that is opposite of what is observed. Thus, we find that the detailed evolution of the X-ray emission from XTE J1810-197 further supports the assumption of a purely thermal spectral model, and leaves no evidence of a steep power law as is commonly fitted to individual observations of AXPs.

Since the first observation of XTE J1810-197 it was apparent that the broad-band pulse shape is not a simple sine function; the pulse peak is relatively sharp with a broader inter-pulse trough (Paper I). This effect is more pronounced at higher energy, where the profile is nearly triangular in shape. This suggests that the pulse profiles can be decomposed into a triangle function and a sinusoidal function, a model that was explored for the earlier data sets and detailed in Paper II \& III. This model continues to be appropriate for the new data and is used to extract unbiased pulsed fraction measurements for the profiles shown in Figure 6 .

Given the success in modeling the pulse profiles with these functions it is natural to associate the two pulsed components uniquely with the two spectral components, i.e., the triangle shape for the hotter blackbody component and the sinusoidal shape for the cooler one. However, we find that we can not model all the profiles in a consistent manner with just a simple superposition of these temporal components, based on the implied flux ratio in each energy band (Gotthelf \& Halpern 2005).
Instead we conclude that either the two spectral components contain an admixture of the two shapes or there is a third, unmodeled spectral component present. This is a direction of active research, to consider the correct characterization of the phase dependent spectral contribution.

\section{Radio observations of XTE J1810-197}

Considering that the absence of radio emission is a defining characteristic of AXPs, the serendipitous radio detection from XTE J1810-197 came as a great surprise. A chance search of VLA data taken about a year after outburst reveals an unresolved point source with a flux of $4.5 \mathrm{mJy}$ at $1.43 \mathrm{GHz}$, located at the precise coordinates of the TAXP (Halpern et al. 2005b). Other archival VLA data obtained at various frequencies provide upper-limits before and after outburst. A follow-up VLA observation in 2006 March yielded a flux of $12.9 \mathrm{mJy}$ at $1.43 \mathrm{GHz}$. Together, these results indicate highly erratic radio emission. A second surprise came with the detection around that time of pulsed radio emission at the $\mathrm{X}$-ray period (Camilo et al. 2006). This search, at $\nu=1.4 \mathrm{GHz}$ using the Parkes radio observatory, revealed a narrow, bright pulse with a high degree of linearly polarization (Camilo et al. 2006). A series of multi-frequency measurements in 2006 April-May confirms the erratic flux behavior and provides an unusual spectral slope of $\alpha \gtrsim-0.5$, where $S_{\nu} \propto \nu^{\alpha}$ (cf. $\alpha \sim-1.6$ for a typical radio pulsar). A revised distance to the pulsar of $3.3 \mathrm{kpc}$ is inferred from the pulsar's dispersion. A search for pulsations in archival data acquired in 1997/1998 produced a null result and argues against significant emission prior to the outburst event. A summary of these radio observations is shown in Figure 7.

\section{Emission Geometry: Models \& Theory}

Although transient AXPs such as XTE J1810-197 are relatively rare, their short active duty cycle suggests the existence of a larger population of unrecognized young NSs. XTE J1810-197 provides a unique window into this population, with prior measurements in the quiescent state and detailed observations during its active, pulsed phase. Ultimately, we hope to use this TAXP to give insight into the emission mechanisms of magnetars, in general. With the discovery of pulsed radio flux, this may carry over to interpreting emission mechanism of radio pulsars, as well. Below we now summarize our initial attempts to interpret the XTE J1810-197 results in the context of a physical model. First we outline the basic arguments for the two-temperature blackbody spectral model as a plausible alternative to the nominal powerlaw plus blackbody model. 


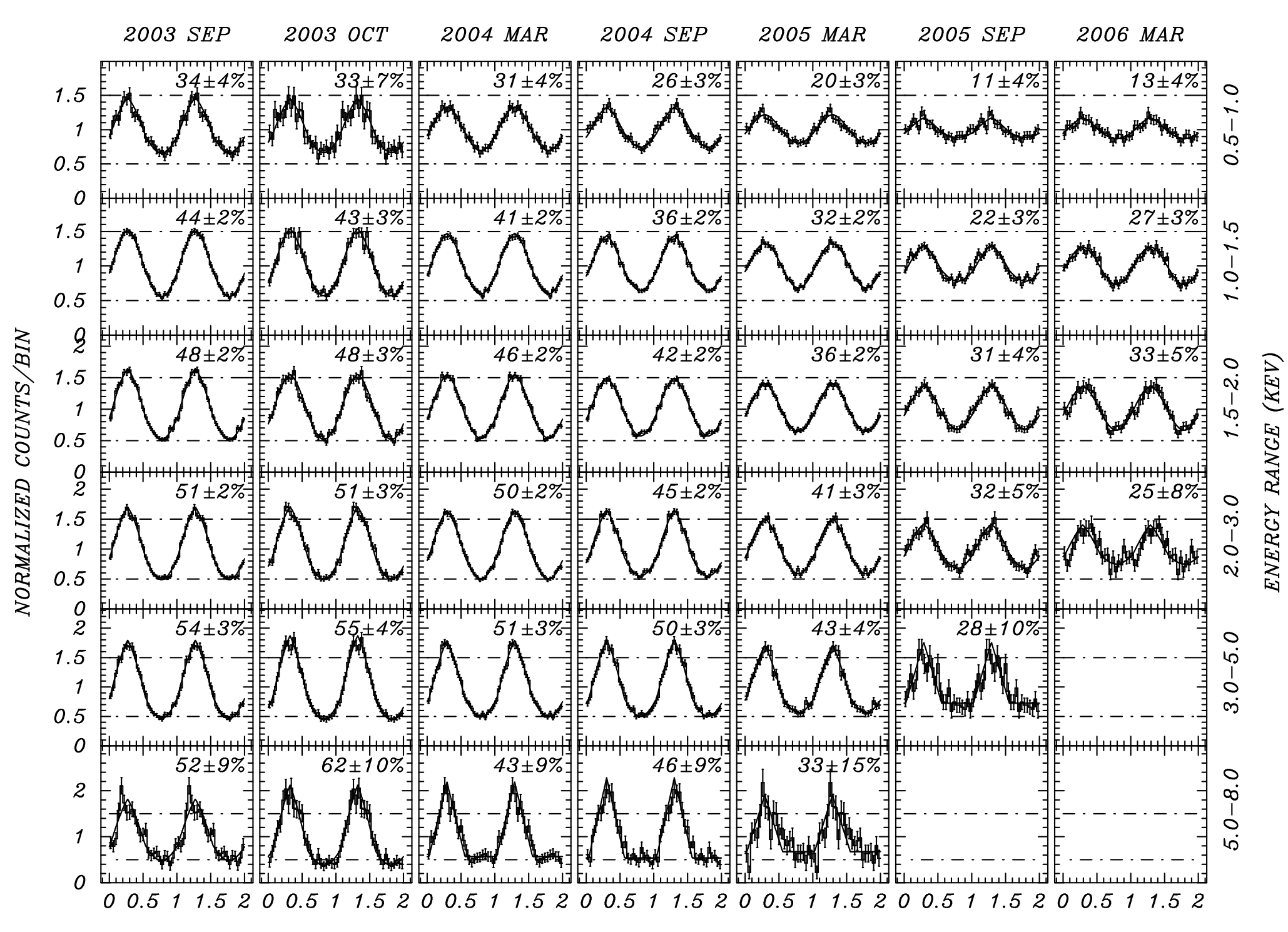

PHASE

Fig. 6 Energy-dependent pulse profiles of XTE J1810-197 obtained with the XMM-Newton EPIC pn detector for all seven epochs of Table 1. The background for each profile has been subtracted and phase zero is aligned for each epoch, for comparison. The phase of the peak is seen to be energy independent. The pulsed fraction at low X-ray energies has decreased with time, while remaining essentially unchanged at high energy. Also shown is the best fit to the two-component model for the pulse profile (solid line) described in the text (see $\$ 2.1$ ). 


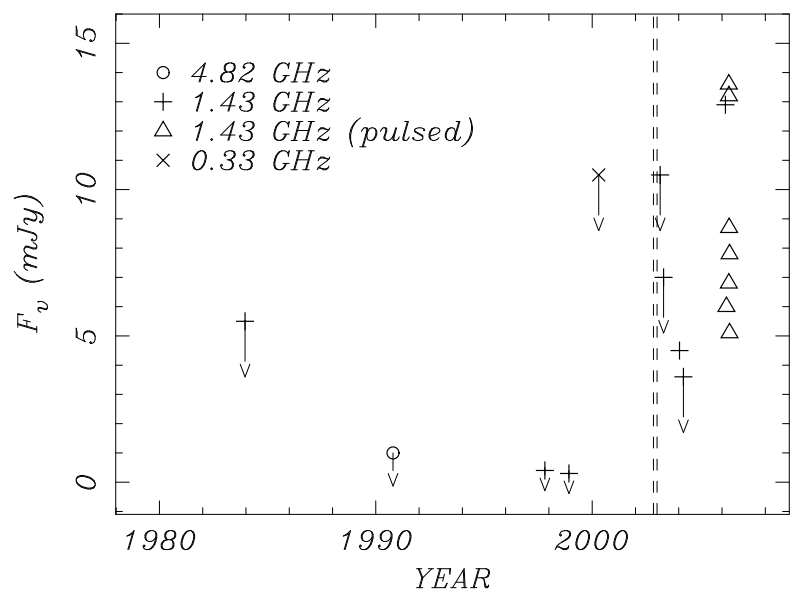

Fig. 7 A set of archival and dedicated radio observations of XTE J1810-197 measured at three frequencies. Prior to outburst (denoted by vertical lines) no radio emission is found corresponding to the plotted upper-limits. Following the Xray outburst, large variations in flux are inferred from several measurements and upper-limits. Pulsed radio emission is also recorded at the X-ray period in the post-outburst era following the 2006 discovery. A search of archival data prior to the outburst places an upper-limit of $\sim 0.4 \mathrm{mJy}$ at $1.4 \mathrm{GHz}$. For clarity, multi-frequency measurements coinciding with the $1.4 \mathrm{GHz}$ pulsed emission data points are not shown (from Halpern et al. 2005b; Camilo et al. 2006).

While both models give reasonable fits to the spectrum, it's important to note that the power-law component is used to model the low energy residuals and not any high-energy tail. Without a low energy cut-off of this component, it is not possible to connect it with the observed IR emission without an energy catastrophe. As detailed in Paper II, invoking synchrotron self-absorption is excluded by radius/magnetic-field inconsistently. In contrast, the extrapolated spectrum of the warm blackbody component does not exceed the measured IR flux. Furthermore, currently there is no acceptable physical model to anchor a power-law component. As discussed earlier, the two components of the double blackbody model can be associated with a pair of hot spots on the surface of the star. The warm temperature component, covering a large fraction of the NS surface, is consistent with the decreased pulsed fraction at lower energies. The hotter blackbody component is consistent with a smaller emission area and greater modulation at higher energies. A comprehensive discussion of this issue can be found in paper II.

Determining the emission geometry on the NS is of great interest. The natural conclusion of applying the double blackbody model to the time resolved spectra and pulse profiles is that concentric hot regions give rise to the observed modulation. We consider a model of the phase-resolved spectrum taking into account the viewing geometry and offset of the hot spots from the rotation axis (Perna \& Gotthelf 2006, in prep.). This is based on the NS emission model given in Perna et al. (2001) that includes general relativistic effects (redshift and light bending). Our goal is to match spectrum and energy dependent pulse shape in order to determine viewing geometry, distance, and NS size. Preliminary work is able to reproduce the pulsed fraction to a reasonable degree but the pulse shape remains elusive.

A framework for a theoretical interpretation of the emission from XTE J1810-197 is suggested by the magnetar coronal model of Beloborodov \& Thompson (2006). According to this model, the large outburst was generated by a starquake that resulted in a transition to an active coronal state that caused energy to be stored in the twisted B-field of the coronal loop. Particles (mostly $e^{+} e^{-}$) are accelerated within this loop and impact the NS surface with GeV energy. This heats up the loop footprint resulting in the observed hot spot emission. The decay timescale of the coronal loop, and thus the hotter component of the double blackbody luminosity, is of order of a few years. The decay rate is determined by ohmic dissipation of current in the excited loop. A cooler component likely arises from deep crustal heating associated with the initial outburst and possibly earlier ones. The features of this model are in general accordance with the observational properties and inferred model for XTE J1810-197.

Acknowledgements We thank Fred Jansen and Norbert Schartel for providing the four XMM-Newton Targets of Opportunity observations of XTE J1810-197.

\section{References}

Beloborodov, A., M. \& Thompson, C. ApJ, in press (2006); arXiv:astro-ph/0602417

Camilo, F., Ransom, S., Halpern, J., et al. Nature, in press (2006); astro-ph/0605429

Duncan, R. C., \& 'Thompson, C. ApJL, 392, L9 (1992)

Gotthelf, E. V., Halpern, J. P., Buxton, M., et al. ApJ, 605, 368 (2004); Paper I

Gotthelf, E. V. \& Halpern, J. P. ApJ, 632, 1075 (2005); Paper III

Halpern, J. P. \& Gotthelf, E. V. ApJ, 618, 874 (2005); Paper II

Halpern, J. P. \& Gotthelf, E. V., Becker, et al. ApJ, 632, L29 (2005)

Ibrahim, I. A., et al. ApJL, 609, L21 (2004)

Kaspi, V. M., Gavriil, F. P., Woods, P. M., et al. ApJ, 588, $93(2003)$

Mereghetti, S., Chiarlone, L., Israel, G. L., et al. Proc. of the 270. WE-Heraeus Seminar, 29 (2002)

Özel, F. ApJ, 563, 276 (2001)

Özel, F., Psaltis, D., \& Kaspi, V. M. ApJ, 563, 255 (2001)

Perna, R., Heyl, J. S., Hernquist, L. E., et al. ApJ, 557, 18 (2001)

Rea, N., et al. Astron. Telegram, 284, 1 (2004a)

- AAP, 425, L5 (2004b)

Thompson, C., \& Duncan, R. C. ApJ, 473, 322 (1996)

Woods, P. M., Kouveliotou, C., Gavriil, F. P., et al. ApJ, 629, 985 (2005) 